\section{perifèria}

Número 18(2), diciembre 2013

http://revistes.uab.cat/periferia

\title{
El uso de los recursos leñosos entre grupos cazadores-recolectores patagónicos: Estudio de la movilidad a partir de la arqueobotánica
}

\author{
Laura Caruso Fermé - Universitat Autònoma de Barcelona ${ }^{1}$
}

\section{Resumen}

Tradicionalmente, en arqueología, el conocimiento de los territorios de captación ha sido caracterizado a partir de la determinación de la procedencia de las materias primas abióticas. Sin embargo, material leñoso también pueden ofrecer datos sobre los las estrategias de movilidad de un grupo. Los resultados obtenidos en el sitio Orejas de Burro 1 (Santa Cruz, Argentina) permiten sugerir distintas vías de circulación en relación a la adquisición de la madera: "interior-costa" y "hacia o desde la cordillera de los Andes".

Palabras claves: recursos leñosos, estrategias de movilidad, cazadoresrecolectores, Patagonia.

\begin{abstract}
Traditionally, in archeology, knowledge about catchment areas has been characterized through the identification of abiotic raw material sources. However, woody material can provide data about mobility strategies of groups. The results from the archaeological site Orejas de Burro 1 (Santa Cruz, Argentina) allow us to suggest different ways of movement related with wood acquisition: "inland - coast" and "out and from the Andes".
\end{abstract}

Keywords: wood resources, mobility strategies, hunter-gatherers, Patagonia.

\section{Introducción}

En todos los tipos de formaciones vegetales -arbóreas, arbustivas, matorrales- se encuentran materias naturales susceptibles de convertirse en recursos utilizables por los integrantes de cualquier tipo de sociedad. Las especies leñosas son recursos en tanto se seleccionan para satisfacer necesidades sociales concretas tales como: iluminación, cocción de alimentos, fabricación de instrumentos, entre otras. Desde los inicios de la humanidad el aprovechamiento de la madera ha sido fundamental

\footnotetext{
${ }^{1}$ Laboratori d'Arqueobotanica. Departament de Prehistòria, Universitat Autònoma de Barcelona Enviar correspondencia a: Laura Caruso Fermé (Icarusoferme@gmail.com)
} 


\section{perifèria}

Número 18(2), diciembre 2013

http://revistes.uab.cat/periferia

para la supervivencia y se han implementado estrategias de subsistencia encaminadas a su explotación. El uso de este recurso se documenta prácticamente desde los orígenes de la humanidad (Keeley, 1981), si bien no es hasta que se generaliza el uso del fuego que encontramos de manera recurrente restos de madera carbonizada en los yacimientos arqueológicos (Dillehay, 1990; Goren-Inbar et al., 2004).

La oferta y distribución de las especies leñosas en el ambiente puede ser altamente variable: continua o discontinua, parejamente dispersa o en parches, abundante o escasa. Por esta razón es posible encontrar formaciones vegetales de tipo forestal, esteparia, ecotono bosque-estepa, etc. La gestión de los recursos leñosos por parte de una sociedad, se encuentra condicionada por diversos factores (disponibilidad y abundancia de la especies en el entorno, etc.). Sin embargo, la funcionalidad, duración de ocupación de un sitio y la complejidad o grado de organización del grupo (grado de movilidad) son elementos que interactúan y a la vez condicionan las modalidades de adquisición y uso de los recursos vegetales leñosos. El estudio arqueobotánico posibilita un acercamiento a aquellas actividades que pudieran estar relacionadas con la gestión de los recursos vegetales por parte de los distintos grupos del pasado. Conocer el papel que jugaron estos recursos dentro de una sociedad brindará, por un lado, una mejor aproximación a la dinámica social y económica de un grupo. Por otro, información de carácter paleoambiental.

Tradicionalmente, en arqueología, el conocimiento de los territorios de captación y redes de intercambio se ha visto limitado por el hecho de que tan sólo han sido caracterizados a partir de la determinación de la procedencia de las materias primas abióticas. Sin embargo, el material leñoso también puede ofrecer datos sobre los diferentes territorios de captación y las estrategias de movilidad. Distintos estudios etnográficos (Nicholson, 1981; Gorecki, 1991; Walthall, 1998; etc.) demuestran que la obtención del combustible se lleva a cabo en las cercanías de los asentamientos. Siguiendo los modelos de área de forrajeo o foraging area, los radios de recolección según los datos etnográficos se sitúan entre 6 y $10 \mathrm{~km}$ desde los lugares de residencia (Binford, 1982), donde entre otros recursos tendría lugar la recolección de la leña. Son numerosos los ejemplos que muestran en los 


\section{perifèria}

Número 18(2), diciembre 2013

http://revistes.uab.cat/periferia

conjuntos arqueobotánicos la presencia de plantas procedentes de ambientes alejados o que son incoherentes con las características ambientales, según criterios actualistas, de la mayoría de los restos arqueobotánicos. Es decir que los conjuntos arqueobotánicos pueden reflejar los diferentes territorios de captación como resultado de los patrones de movilidad del grupo y de sus miembros. En este sentido no podemos asumir que todos los restos arqueobotánicos procedan de los alrededores del yacimiento. Sin duda la función para la que fueron adquiridos puede ayudar a definir propuestas sobre las áreas de procedencia de las especies. Así, mientras las maderas recolectadas para su utilización como combustible pueden ser de provisión local, dado su uso cotidiano, las maderas recolectadas para otras funciones (instrumentos) no tienen por qué responder a este patrón y su lugar de procedencia puede estar bastante alejado. Es preciso señalar, que los objetos de madera que caen en desuso pueden ser arrojados al fuego y utilizados como combustible aunque esta no fuese la función para la que fueran recolectadas estas especies. Por lo tanto, a partir del análisis taxonómico de los carbones, no se puede determinar si estos responden a una recolección primaria o secundaria. Por otra parte, cabe ser prudente en la extrapolación hacia el pasado de las actuales áreas de dispersión de las distintas especies vegetales. Es importante recordar que los paisajes actuales son el resultado de factores ambientales y sociales y que las distintas disciplinas que tratan de reconstruir la vegetación del pasado no permiten conocer con suficiente grado de detalle las áreas de distribución de las especies.

El presente trabajo se centra en el estudio de las modalidades de adquisición y uso del material leñoso y estrategias de movilidad entre grupos cazadores-recolectores patagónicos (Argentina). Con este propósito se llevó a cabo el análisis arqueobotánico de los materiales -carbón y madera sin carbonizar- procedente del sitio arqueológico Orejas de Burro 1 localizado en el Campo Volcánico Pali Aike, provincia de Santa Cruz (Patagonia, Argentina) -figura 1-. 


\section{perifèria}

Número 18(2), diciembre 2013

http://revistes.uab.cat/periferia

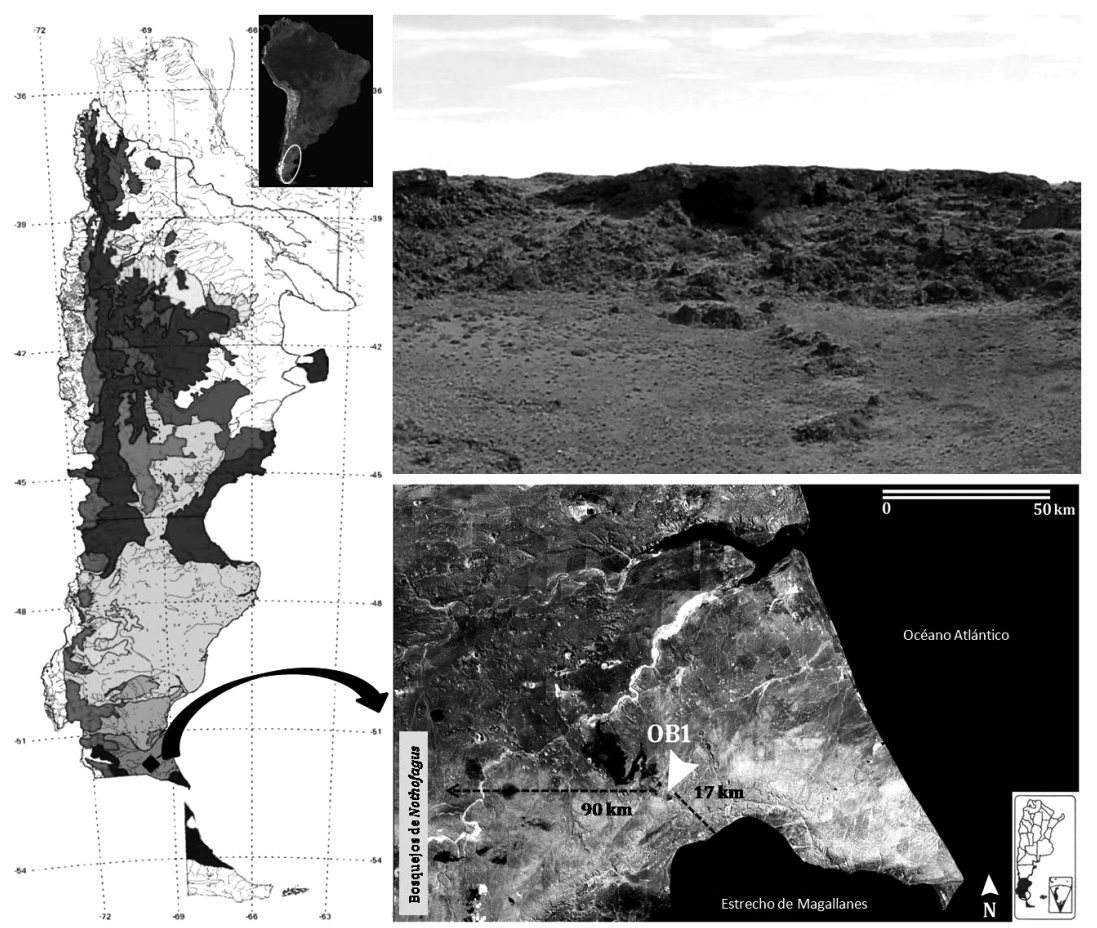

Figura 1: Localización del sitio arqueológico Orejas de Burro 1

\section{El sitio Orejas de Burro y el estudio de la movilidad a partir de la arqueobotánica}

El sitio Orejas de Burro 1 (en adelante OB1) -ca. 3.500 años 14C AP y ca. 1.700500 años 14C AP- se localiza en una cueva ubicada en el interior de un cono volcánico del Campo Volcánico Pali Aike (prov. de Santa Cruz), a 17 km al norte del Estrecho de Magallanes. La estructura geomorfológica de la región de Pali Aike define la movilidad a través de los lugares de paso. La tendencia a la utilización de afloramientos volcánicos creó importantes condiciones para la circulación humana en esta zona de la Patagonia (Borrero y Charlin, 2010). El elevado número de moluscos, registrado en OB1, que carecen de modificaciones antrópicas, sugiere su adquisición para fines alimenticios. Los eventos de transporte articulados en una sola instancia de movimiento, han permitido interpretar que las distancias que separan este sitio de la costa se encuentran dentro del radio de predación regular esperado para grupos cazadores-recolectores (Barberena, 2008). Por otra parte las evidencias isotópicas también aportan información que permite avalar un patrón de movilidad que implica la interacción periódica costa-interior de los grupos 


\section{perifèria}

Número 18(2), diciembre 2013

http://revistes.uab.cat/periferia

cazadores-recolectores que ocuparon esta cueva (Borrero y Barberena, 2006).

Los resultados arqueobotánicos (Caruso Fermé, 2012, 2013; Caruso Fermé y Aviles, 2013, Caruso Fermé y L'Heureux, 2013) evidencian la presencia de tres especies vegetales: Berberis sp., Escallonia rubra -siete camisas- y Nothofagus pumilio -lenga-, la primera de ellas con una casi absoluta representación. Escallonia rubra y Nothofagus pumilio son dos taxones que reflejan una formación vegetal tipo forestal, dominada por especies arbóreas y arbustivas características del actual Bosque caducifolio. Este tipo de formación vegetal es muy diferente a la propia de la Estepa Húmeda, que actualmente caracteriza la zona del Campo Volcánico Pali Aike -lugar donde se localiza OB1-. Los indicadores paleoclimáticos utilizados en la reconstrucción de la historia paleoambiental del Campo Volcánico Pali Aike tampoco evidencian la presencia de estas dos especies en las inmediaciones del sitio arqueológico durante el transcurso del Holoceno (Zolitschka et al., 2006). Cabe mencionar que actualmente el área de localización OB1 está separada aproximadamente por $100 \mathrm{~km}$ de los principales bosquejos de Nothofagus. Los estudios polínicos brindan valiosa información sobre la existencia de formaciones boscosas en el pasado, pero no de sus límites o localización estricta. Por lo tanto, si bien es posible determinar la distancia del sitio a la costa del Estrecho de Magallanes $(17 \mathrm{~km})$, no se puede establecer cuán distantes estaban las zonas boscosas en el momento de ocupación del mismo. La imposibilidad de establecer una distancia aproximada hacia formaciones vegetales de tipo forestal y las evidencias antracológicas obtenidas no permiten plantear que los restos de carbón de Nothofagus pumilio y maderas de Escallonia rubra pudieran estar contenidas dentro de un área integrada en forma logística. Por otra parte, el estudio del material leñoso demostró también modificaciones en la estructura anatómica de algunos de los carbones, similares a las producidas por la inmersión de la madera en agua (Schweingrüber, 1978).

Los resultados obtenidos abrieron el debate en cuanto a la procedencia del material leñoso recuperado en Orejas de Burro 1. Prospecciones realizadas en la costa del Estrecho de Magallanes demostraron que actualmente las corrientes marinas transportan troncos de Nothofagus pumilio. Si se tiene en cuenta la distancia que 


\section{perifèria}

Número 18(2), diciembre 2013

http://revistes.uab.cat/periferia

hoy en día separa el área de localización OB1 de los principales bosquejos de Nothofagus no se descarta la posibilidad de que los desplazamientos hacia la costa marina estuvieran relacionados con la presencia de esta especie en la estratigrafía del yacimiento. Con el objetivo de identificar posibles rasgos que permitieran discriminar restos antracológicos flotados de restos no flotados, se llevó a cabo un programa experimental. El análisis de la composición química de partículas sedimentarias y precipitados minerales de muestras actuales de madera de Nothofagus pumilio y Berberis sp. de distintas procedencias geográficas (bosques y costas de la Patagonia argentina y chilena) y en diferente estado de depositación (sumergida en agua de mar, dulce o directamente árboles en pie) posibilitó determinar diferencias en la composición química de estas mismas especies recuperadas en OB1.

Los distintos análisis realizados aportaron valiosa información que permiten vincular los territorios del interior y la costa. Sin descartar que efectivamente los bosques cordilleranos puedan haber constituido las áreas fuente de los recursos leñosos del sitio, es posible considerar una explicación alternativa sobre la presencia de carbones de Nothofagus pumilio. La adquisición de esta especie leñosa pudo haberse realizado conjuntamente o en el marco de otras actividades realizadas en el espacio costero. Los muestreos efectuados en la costa del Estrecho de Magallanes permitieron verificar que actualmente no presenta restricciones importantes para el acceso al mar y sus recursos y que las corrientes marinas y la morfología de sus costas posibilitan la acumulación de especies leñosas en las playas. La presencia de halita -sal- en algunos restos de material leñoso arqueológico es un claro ejemplo del desarrollo de actividades relacionadas con agua marina. Estos resultados conjuntamente con los altos porcentajes de calcita y la presencia de arcillas en los carbones arqueológicos de Nothofagus pumilio y los restos marinos que vinculan a los ocupantes de OB1 con el Estrecho de Magallanes han permitido proponer un posible uso de madera arrojada por las mareas a la costa del estrecho de Magallanes. Por otra parte tampoco se puede descartar que se hayan utilizado recursos de los ambientes cordilleranos, ya que algunas de las especies presentes en el registro arqueobotánico son típicas de formaciones boscosas. Por lo tanto, los resultados obtenidos hasta el momento permiten sugerir 


\section{perifèria}

Número 18(2), diciembre 2013

http://revistes.uab.cat/periferia

otra vía de circulación además de la ya citada interior-costa (atestiguada por la presencia de moluscos, restos óseos de aves y estudios isotópicos). Esta otra ruta iría "hacia o desde la cordillera de los Andes", donde la diversidad de especies leñosas es más amplia en comparación con la zona donde se encuentra OB1.

\section{Conclusión}

El entorno vegetal no sólo fue esencial en el quehacer cotidiano de los cazadoresrecolectores, sino que los recursos que proporciona tienen el mismo grado de importancia que cualquier otro recurso aprovechado. El estudio del material leñoso recuperado en sitios arqueológicos es una valiosa fuente de información para poder comprender la relación entre los integrantes de los distintos grupos que poblaron la Patagonia y el entorno natural.

El estudio de los restos de carbón y madera sin carbonizar procedentes de Orejas de Burro 1 permitió, por un lado, conocer las especies vegetales leñosas consumidas por los ocupantes de este sitio. Por otro, conocer las modalidades de adquisición y consumo de los distintos recursos leñosos. Finalmente, los resultados obtenidos permiten discutir desde una nueva perspectiva la gestión de los recursos leñosos entre grupos cazadores-recolectores patagónicos. El estudio de la movilidad (circulación/exploración) entre los grupos cazadores-recolectores es factible a través de la realización de distintos tipos de análisis en material leñoso carbonizado y sin carbonizar. Sobre la base de la distribución actual de las comunidades vegetales es posible determinar las áreas de procedencia de las especies arqueológicas. No obstante, es preciso recalcar la prudencia que se debe tener en la extrapolación hacia el pasado de las actuales áreas de dispersión de las distintas especies vegetales.

\section{Bibliografía}

Barberena, R. (2008) Arqueología y biogeografía humana en Patagonia meridional. Colección Tesis Doctorales, dirigida por I. Nacuzzi. Sociedad Argentina de Antropología, Buenos Aires. 


\section{revista de recerca i formació en antropologia}

\section{perifèria}

Número 18(2), diciembre 2013

http://revistes.uab.cat/periferia

Binford, L. R. (1982) The archaeology of place. Journal of Anthropological Archaeology, vol.1, no 1, pp. 5-31

Binford, L. R. (1980) Willow smoke and dog's tails: hunter- gatherer settlement systems and archaeological site formation. American Antiquity, vol. 45. no 1, pp. 420.

Binford, L. R. (2001) Constructing Frames of Reference: An analytical Method for Archeological Theory Building Using hunter-Gatherer and Environmental Data sets. University of California, Berkeley.

Borrero, L. y Barberena, R. (2006) Hunther-gatherer home ranger and marine resources. Current Anthropology, vol. 47, n० 5, pp. 855-867

Caruso Fermé, L. (2012) Modalidades de adquisición y usos del material leñoso entre gurpos cazadores-recolectores patagónicos (Argentina). Métodos y técnicas de estudio del material leñoso arqueológico. Tesis de doctorado, julio 2012 (Universitat Autònoma de Barcelona, España).

https://www.educacion.es/teseo/mostrarRef.do?ref $=986004$

Caruso Fermé, L. (2013) Espacios interdisciplinarios en la Arqueobotánica: alcances y aportes para la investigación arqueológica en Patagonia. En: Tendencias teóricometeodologicas y casos de estudio en la arqueología de la Patagonia. Comp: Zangrando, Barberena, Gil, Neme, Giardina, Luna, Otola; Paulides; Slagan y Trivoli. Pp: 271-279.

Caruso Fermé, L. e Iriarte Avilés E. (2013) Análisis de la composición química de partículas y precipitados minerales en material leñoso: madera flotada o no flotada en el sitio Orejas de Burro 1 (Santa Cruz, Patagonia Argentina). Experimentación en arqueología. Estudio y difusión del pasado. Sèrie Monogràfica del MAC, pp. 5764.

Caruso Fermé, L. y L'Heureux, G. L. (2013) El estudio tafonómico de los restos leñosos del sitio Orejas de Burro 1 (Santa Cruz, Argentina). XVIII Congreso Nacional de Arqueologia Argentina. La Rioja, Argentina. 


\section{perifèria}

Número 18(2), diciembre 2013

http://revistes.uab.cat/periferia

Dillehay, T. (1986) The Cultural Relationship of Monte Verde: A late Pleistocene Settlement Site in the Sub-Antarctic Forest of South-central Chile. En: A.Bryan (ed.) New Evidence for the Pleistocene Peopling of the Americas. Orono, Centerb for the Study if the First Americans, pp. 319-337.

Gorecki, P. P. (1991) Horticulturalists as Hunter-Gatherers: Rock Shelter Usage in Papua New Guinea. In Ethnoarchaeological Approaches to Mobile Campsites, edited by C. S. Gamble and W. A. Broismier, pp. 237-262. International Monographs in Prehistory, Ann Arbor, Michigan.

Goren-Inbar, N.; Nira Alperson; Mordechai E. Kislev; Orit Simchoni; Yoel Melamed; Adi Ben-Nun; Ella Werker (2004) Evidence of Hominin Control of Fire at Gesher Benot Ya`aqov, Israel. Science 30 vol. 304 n 5671, pp. 725-727

Keeley, J. E. (1981) Reproductive cycles and fire regimes. In: Mooney, H. A.; Bonnicksen, T. M.;Christensen, N. L.; Lotan, J. E.; Reiners, W. A., eds. Proceedings of the conference fire regimes andecosystem properties. Washington, D.C.: USDA Forest Service, General Technical Report WO-26, pp. 231-277

Nicholson P.H. (1981) Fire and the Australian Aborigine: an enigma, in A.M. Gill et I.R. Noble dir., Fire and the Australian biota, Australian Academy of Science, Canberra, pp. 55-76.

Schweingruber, F.H. (1978). Tree Rings. Dordrecht/Boston/London, Kluwer Academic Publishers.

Walthall, J.H. (1998) Rockshelters and hunter-gatherer adaptation to the Pleistocene/Holocene transition, American Antiquity, vol. 63, no 2, pp. 223-238.

Zolitschka, B.; Schäbitz, F., Lücke, A., Corbella, H.; Ercolano, B.; Fey, M.; Haberzettl, T.; Janssen, S.; Maidana, N.; Mayr, C.; Ohlendorf, C.; Oliva, G., Paez, M.; Soto, J.; Tiberi, P. Y Wille, M. (2006) Schleser, G. Crater lakes of the Pali Aike Volcanic Field as key sites for paleoclimatic and paleoecological reconstructions in southern Patagonia, Argentina. Journal of South American Earth Sciences, vol. 21, pp. 294-309 